\title{
Diagnostic Utility of Bronchial Washing CBNAAT for Mycobacterium Tuberculosis in Sputum Smear Negative and Sputum CBNAAT Negative Patients of Suspected Pulmonary Tuberculosis
}

\author{
Afshan Ali Shaik ${ }^{* 1}$, Uday Kakodkar ${ }^{2}$, Cigy Borges ${ }^{3}$ \\ ${ }^{1}$ Senior Resident, Department of Pulmonary Medicine, Goa Medical College, Taleigao, Goa, India \\ ${ }^{2}$ Associate Professor and Unit Head, Department of Pulmonary Medicine, Goa Medical College, Taleigao-Goa, India \\ ${ }^{3}$ Microbiologist, Department of Microbiology, Goa Medical College, Goa, India \\ *Corresponding author: Afshan Ali Shaik; Chest Diseases and Tuberculosis Hospital, PO Caranzalem, Tiswadi, Goa; \\ afshanshaik68@gmail.com
}

Received 17 February 2021;

Accepted 07 March 2021;

Published 16 March 2021

\begin{abstract}
Introduction: Diagnosis of sputum negative pulmonary tuberculosis can be challenging and time consuming, with many patients being put on empirical anti-tuberculosis treatment. Bronchoscopy helps in early diagnosis in such patients. Aims and Objectives: To assess the diagnostic utility of bronchial washing CBNAAT for Mycobacterium Tuberculosis in sputum smear negative and sputum CBNAAT negative patients of suspected pulmonary tuberculosis. Methodology: This case series was conducted on 71 patients in the Department of Pulmonary Medicine, Goa Medical College from May 2018- February 2020. Patients with suspected pulmonary tuberculosis on chest radiograph and/or CT thorax, but with sputum smear as well as CBNAAT for Mycobacterium Tuberculosis negative were subjected to flexible video bronchoscopy. Bronchial washings were collected from the affected lobes and the specimen were subjected to fluorescent microscopy, CBNAAT, MGIT, bacterial and fungal culture. Results: The diagnosis of tuberculosis was established in 23 (32.4\%) patients in bronchial washings specimen by CBNAAT. These patients were followed up after 6 months of anti-tuberculosis treatment and have shown significant clinical and radiological improvement. Conclusion: Bronchoscopy is a useful tool in diagnosis of pulmonary tuberculosis in sputum negative patients. It also play an important role in diagnosis of other infectious /malignant disorder which can mimic tuberculosis.
\end{abstract}

Keywords: bronchoscopy, sputum smear PTB, CBNAAT

\section{Introduction}

About one fourth of the world's population is estimated to be infected with M.tuberculosis ${ }^{[1,2]}$. On an average, about $5-10 \%$ of those infected will develop tuberculosis disease over their course of lives and most of them will develop TB within the first five years after initial infection ${ }^{[3]}$. Sputum negative pulmonary disease remains a major challenge due to under recognition,under diagnosis and poor management of such cases. It is estimated that smear negative pulmonary tuberculosis accounts for $30-60 \%$ of cases of pulmonary tuberculosis and is one of the cause for high mortality in HIV patients ${ }^{[4]}$. If these patients are untreated then about $70 \%$ of them may develop active tuberculosis in a year ${ }^{[5,6]}$. Bronchoscopy plays an important role in the microbiological confirmation of tuberculosis in smear negative patients. Flexible
Video Bronchoscopy can also help in diagnosis of several lung conditions which can mimic pulmonary tuberculosis ${ }^{[7]}$.

\section{Aims and Objectives}

1. To assess the diagnostic utility of bronchial washing CBNAAT for Mycobacterium Tuberculosis in patients suspected to have pulmonary tuberculosis who were sputum smear negative and sputum CBNAAT negative.

2. To confirm diagnosis of pulmonary tuberculosis in sputum negative pulmonary tuberculosis.

3. To assess the role of bronchoscopy in early diagnosis of sputum negative pulmonary tuberculosis and to rule out other diseases like pneumonia and malignancy. 


\section{Methodology}

This case series was conducted on 71 patients in the Department of Pulmonary Medicine, Goa Medical College from May 2018February 2020.Institutional ethics committee approval was obtained in the present study.

The inclusion criteria were 1) New sputum negative pulmonary tuberculosis patients above 12 years of age with chest radiograph/CT thorax suggestive of active pulmonary tuberculosis.2) patients whose induced sputum smear microscopy and CBNAAT sample was negative for tuberculosis.

Exclusion criteria 1) Who were previously treated patients for pulmonary Tuberculosis 2) who had contraindication for bronchoscopy like patients with recent myocardial infarction, unstable angina, arrhythmias, severe bleeding disorder.

All inpatients and outpatients who were presumptive TB suspects (as per NTEP definition) were subjected to sputum smear microscopy, CBNAAT and Chest X-ray. Sputum CBNAAT testing for Mycobacterium Tuberculosis was conducted, in patients who were sputum smear negative but had Chest X-ray suggestive of PTB or in patients who had high clinical suspicion for Tuberculosis. In patients with dry cough / with scanty sputum or in patients who are unable to expectorate and with high clinical suspicion of PTB, sputum was induced using 3\% hypertonic saline. This induced sputum was subjected to smear microscopy and CBNAAT.

Only in those cases with high clinical suspicion for tuberculosis, CT thorax was considered if sputum smear and CBNAAT is negative. If CT thorax findings were normal than the patients were excluded from the study. Sputum negative patients (with induced smear microscopy and CBNAAT negative) with symptoms suggestive of pulmonary Tuberculosis were subjected to bronchoscopy.

Bronchial washings were obtained by instillation of $30 \mathrm{ml}$ $40 \mathrm{ml}$ of $0.9 \%$ saline into the segment of an affected lobe. The segment chosen for doing bronchial washings was selected based on Chest X-Ray PA and Lateral view or based on CT thorax (in majority of patients).These bronchial washings were subjected to smear microscopy by ZN stain, CBNAAT, MGIT, bacterial culture and fungal culture. Bronchial biopsies were obtained in 4 cases where lesions were suspicious of a non-tubercular etiologies. The data was analyzed using SPSS software.

\section{Results}

There were 46(64.8\%) males and 25(35.2\%) females in our present study.Most patients were in the age group of 18-72 years.

The common Chest X-ray findings seen in our patients were infiltration in $26(36.6 \%)$, consolidation in $25(35.2 \%)$, cavitation in $18(25.4 \%)$, loss of volume in $10(14.1 \%)$ and effusion in $4(5.6 \%)$ of cases.

HRCT thorax was done in $57(80 \%)$ patients. The common findings seen were centrilobular nodules in tree and bud appearance in 39(54.9\%), consolidation in $25(35.2 \%)$, cavitation in $24(33.8 \%)$, mediastinal lymphadenopathy in 18 (25.4\%), collapse in $12(16.9 \%)$, pleural effusion in $7(9.9 \%)$, intrabronchial abnormalities in $3(4.2 \%)$ cases.

The most common bronchoscopy findings seen in our CBNAAT positive cases were normal tracheobronchial tree in 14 $(60.8 \%)$ cases followed by intrabronchial mucopurulent secretions in $7(30.4 \%)$, intrabronchial growth in $5(21.7 \%)$,ulceration in 4 $(17.3 \%)$, hyperemia in $2(8.6 \%)$ and intrabronchial narrowing in 2 $(8.6 \%)$ of cases.

CBNAAT for Mycobacterium tuberculosis was detected in $23(32.3 \%)$ and this patients were initiated on AKT. Out of the remaining $48(66.2 \%)$ non tuberculous patients, $10(14 \%)$ had bacterial infection (suppurative lung disease), I (1.4\%) had fungal lung disease and $2(2.8 \%)$ had bronchogenic carcinoma. One patient had superadded bacterial infection with Serratia along with Tuberculosis.

Table 1: Diagnostic yield of flexible video bronchoscopy

\begin{tabular}{|l|l|l|}
\hline Bronchial washings & Positive report (no) & $\%$ \\
\hline 1) AFB smear & 11 & $15.5 \%$ \\
\hline 2)CBNAAT & 23 & $32.4 \%$ \\
\hline 3) MGIT culture & 22 & $38.6 \%$ \\
\hline 4) Bacterial infection & 11 & $15.5 \%$ \\
\hline 5) fungal & 1 & $1.4 \%$ \\
\hline 6) Malignancy & 2 & $2.8 \%$ \\
\hline Total & 71 & \\
\hline
\end{tabular}

Table 2: Efficacy of bronchial washings AFB smear versus CBNAAT and MGIT in the diagnosis of pulmonary tuberculosis

\begin{tabular}{|l|l|l|l|l|l|}
\hline \multirow{2}{*}{ AFB Smear } & Positive CBNAAT & Positive MGIT & \multirow{2}{*}{ P value } \\
\cline { 2 - 6 } & Number(n)/total & \% & Number (n)/total & \% & \\
\hline Positive smear & $11 / 71$ & $15.5 \%$ & $11 / 57$ & $19.3 \%$ & 0.00 \\
\hline Negative smear & $12 / 71$ & $16.9 \%$ & $11 / 57$ & $19.3 \%$ & 0.00 \\
\hline
\end{tabular}

Table 3: Validity of bronchial washings CBNAAT as compared to AFB smear

\begin{tabular}{|l|l|l|l|l|l|}
\hline Test on bronchial washings & Frequency and percentage n (\%) & Sensitivity & Specificity & PPV (\%) & NPV(\%) \\
\hline CBNAAT for MTB & $23(32.4 \%)$ & $100 \%$ & $80 \%$ & $47.8 \%$ & $100 \%$ \\
MTB detected & $48(67.6 \%)$ & & & & \\
MTB not detected & & & & \\
\hline
\end{tabular}




\section{Discussion}

In the earlier days prior to the advent of bronchoscopy, patients with smear negative pulmonary tuberculosis were empirically started on anti-tuberculosis treatment based on the chest radiograph findings. This could result in over diagnosis of tuberculosis and missing some of the infectious or malignant conditions which could mimic tuberculosis in its clinical or radiological presentation. With the advent of bronchoscopy, CBNAAT or smear for mycobacterium from bronchial washings, bronchoalveolar lavage fluid or bronchial brushings has been used for the diagnosis of pulmonary tuberculosis.

In present study, the most common bronchial findings seen in $\mathrm{TB}$ cases were normal tracheobronchial tree in $14(60.8 \%)$ followed by intrabronchial mucopurulent secretions in 7(30.4\%), intrabronchial growth in $5(21.7 \%)$, ulceration or erosions in 4 $(17.3 \%)$, congestion with hyperemia of bronchial mucosa in 2 $(8.6 \%)$ and intrabronchial narrowing in $2(8.6 \%)$ of cases. This findings are similar to findings found by Sharma et al which shows mucopurulent secretions in $37.2 \%$, congestion in $32.5 \%$, normal tracheobronchial tree in $25 \%$ as common findings ${ }^{[8]}$.

The smear examination of the Bronchial washings detected AFB in $11(15.5 \%)$ patients as shown in table 1. In a similar study done by Vikas Gupta et al, the diagnostic yield was established in $21(45.7 \%)$ patients ${ }^{[9]}$. Bronchial washings for CBNAAT and MGIT in our study as shown in table 1 gave the diagnostic yield in $23(32.4 \%)$ and $22(38.6 \%)$ patients respectively. The lower diagnostic yield and lower efficacy of AFB smear as compared to CBNAAT /MGIT as shown in table 2 could be explained by the fact that about 5,000-10,000 acid fast bacilli (AFB) per $\mathrm{ml}$ of specimen must be present for detection by smear whereas culture requires 10-100 viable organisms ${ }^{[10,11]}$ and CBNAAT requires 131 $\mathrm{CFU} / \mathrm{ml}$ of specimen ${ }^{[12]}$. The limitation of this study was due to insufficient sample obtained, liquid culture could be done of around 57 patients as the same was not available in the initial part of the study.

Bronchial washings for CBNAAT in our study gave the diagnostic yield in $23(32.4 \%)$ as shown in table 1 patients and all our patients were Rifampicin sensitive. This yield is comparable to study done by Le Palud et al where the diagnostic yield was 18(37.5\%) ${ }^{[13] .}$ The advantage of CBNAAT assay over AFB smear was that CBNAAT would in addition gives the rifampicin resistance /sensitivity status of the patient. The results by MGIT 960 medium takes nearly 42 days whereas CBNAAT results are ready in 2 hours, hence CBNAAT is a better tool for early diagnosis of patients. Any delay in diagnosis in sputum negative patient especially drug resistant patients can have serious consequence for the patient and the community ${ }^{[14,15] \text {. }}$

Numerous studies have demonstrated the utility of CBNAAT in diagnosis of pulmonary tuberculosis on bronchial washings, the CBNAAT sensitivity varies from $51.9 \%-97.2 \%$ and specificity varies from $73.2-100 \%$ on bronchial washings ${ }^{[13,16-19]}$. The sensitivity in our study as shown in table 3 was $100 \%$ and specificity was $80 \%$. The positive predictive value was $47.8 \%$ and negative predicted value $100 \%$.

\section{Conclusion}

This study thus emphasizes the role of bronchoscopy by CBNAAT in providing a good specimen and early diagnosis of tuberculosis in patients who are Sputum negative but with high clinical and radiological suspicion as shown by our high sensitivity results. In suspected cases of sputum negative pulmonary tuberculosis, the diagnosis of tuberculosis was established in 23 (32.4\%) cases. Bronchoscopy is superior in the differential diagnosis of tuberculosis with other commonly encountered diseases like pneumonia or lung cancer.

\section{Funding}

No funding sources

\section{Conflict of interest}

None

\section{Acknowledgement}

We would like to thank the Dean of Goa Medical College for allowing us to perform this study

\section{References}

[1] Houben RMGJ, Dodd PJ. The global burden of latent tuberculosis infection, a re-estimation using mathematical modeling. PLOS Med.2016; 13(10): e1002152.

[2] Cohen A, Mathiasen VD, SchonT et al.The global prevalence of latent tuberculosis: a systematic review and meta-analysis. Eur Respir J.2019; 54(3):1900655.

[3] Comstock GW, Livesay VT, Woolpert SF. The prognosis of positive tuberculin reaction in childhood and adolescence. AmJ Epidemiol.1974; 99(2):131-8.

[4] Campos LC, Viera Rocha MV, Cunha Willers et al. Characteristics of patients with smear negative pulmonary tuberculosis in a region with high TB and HIV prevalence. PLoS One.2016; 11(1):1-8.

[5] World Health Organization (WHO) :Global tuberculosis report.2013.

[6] Available from: https//apps.who.int/iris/handle/10665/91355.

[7] Hong Kong Chest Service/Tuberculosis Research Centre, Madres/ British Research Council.A controlled trial of a 2-month,3-month, and 12-month regimen of chemotherapy for sputum smear -negative pulmonary tuberculosis: the results at 30 months. Am Rev Respir Dis. 1981; 124(2):138-42.

[8] Araz O, Akgun M, Salgam L et al.The diagnostic value of bronchoscopy in smear negative cases of pulmonary tuberculosis. Tuberk Torak. 2008; 56(2): 150-157.

[9] Sharma Shubhkaran, Luhadia S.K.,Gupta N.K..Clinical evaluation of flexible fibreoptic bronchoscope in diagnosis of new cases of sputum smear negative pulmonary tuberculosis. ASME.2013; 1(2):93-100.

[10] Vikas Gupta, Anurag Agrawal, Lalit Singh et al. The role of bronchoscopy in the diagnosis of sputum smear negative pulmonary tuberculosis.JMSCR.2017; 5(2):17393-17398.

[11] Kim TC, Blackman RS, Heatwle KM et al. Acid-fast bacilli in sputum smear of patients with pulmonary tuberculosis. Am Rev Respir Dis. 1984; 129(2):264-268.

[12] Colebunders R,Bastian J. A review of the diagnosis and treatment of smear negative pulmonary tuberculosis.Int $J$ Tuberc Lung Dis. 2000 ;4(2):97-107.

[13] Helb D,Jones M,Story E,et al.Rapid detection of mycobacterium tuberculosis and rifampicin resistance by 
use of on -demand, near-patient technology.J Clin Microbiol.2010;48(1):229-237.

[14] Le Palud P, Cattoir V,Malbruny B et al.Retrospective observational study of diagnostic accuracy of Xpert MTB/RIF assay on fibreoptic bronchoscopy sampling for early diagnosis of smear negative or sputum-scarce patients with suspected pulmonary tuberculosis. BMC Pulm Med.2014;14: 137.

[15] Sun JR, Lee SY, Perng CL,et al. Detecting Mycobacterium tuberculosis in Bactec MGIT 960 cultures by in house IS6110- based PCR assay in routine clinical practice.J Formos Med Assoc.2009;108(2):11925.

[16] Zhao P,Fang F,Yu Q, et al.Evaluation of Bactec MGIT 960 system for testing susceptibility of Mycobacterium tuberculosis to first -line drugs in China. PLoS One. 2014; 9(9):e99659.
[17] Tamura A, Shimada M, Matsui Y, et al.The value of fibreoptic bronchoscopy in culture-positive pulmonary tuberculosis patients whose pre bronchoscopic sputum specimens were negative for smear and PCR analyses. Intern Med. 2010; 49(2): 95-102.

[18] Min JW,Yoon HI,Park KU,et al. Real -time polymerase chain reaction in bronchial aspirate for rapid diagnosis of sputum smear negative tuberculosis.Int $\mathrm{J}$ Tuberc Lung Dis. 2010;14(7):852-8.

[19] Chen NH,Liu YC,Tsao TC et al. Combined bronchoalveolar lavage and polymerase chain reaction in the diagnosis of pulmonary tuberculosis in smear negative patients. Int J tuberc Lung Dis.2002;6(4) :3505.

[20] Boehme C, Nabeta P, Hillemann D, et al. Rapid molecular detection of tuberculosis and rifampicin resistance.N Eng J Med.2010;363:1005-15. 